\title{
REGULARITY AND H-POLYNOMIALS OF MONOMIAL IDEALS
}

\author{
TAKAYUKI HIBI AND KAZUNORI MATSUDA
}

\begin{abstract}
Let $S=K\left[x_{1}, \ldots, x_{n}\right]$ denote the polynomial ring in $n$ variables over a field $K$ with each $\operatorname{deg} x_{i}=1$ and $I \subset S$ a homogeneous ideal of $S$ with $\operatorname{dim} S / I=d$. The Hilbert series of $S / I$ is of the form $h_{S / I}(\lambda) /(1-\lambda)^{d}$, where $h_{S / I}(\lambda)=h_{0}+h_{1} \lambda+h_{2} \lambda^{2}+\cdots+h_{s} \lambda^{s}$ with $h_{s} \neq 0$ is the $h$-polynomial of $S / I$. It is known that, when $S / I$ is Cohen-Macaulay, one has reg $(S / I)=\operatorname{deg} h_{S / I}(\lambda)$, where $\operatorname{reg}(S / I)$ is the (Castelnuovo-Mumford) regularity of $S / I$. In the present paper, given arbitrary integers $r$ and $s$ with $r \geq 1$ and $s \geq 1$, a monomial ideal $I$ of $S=K\left[x_{1}, \ldots, x_{n}\right]$ with $n \gg 0$ for which $\operatorname{reg}(S / I)=r$ and $\operatorname{deg} h_{S / I}(\lambda)=s$ will be constructed. Furthermore, we give a class of edge ideals $I \subset S$ of Cameron-Walker graphs with $\operatorname{reg}(S / I)=\operatorname{deg} h_{S / I}(\lambda)$ for which $S / I$ is not Cohen-Macaulay.
\end{abstract}

\section{INTRODUCTION}

Let $S=K\left[x_{1}, \ldots, x_{n}\right]$ denote the polynomial ring in $n$ variables over a field $K$ with each $\operatorname{deg} x_{i}=1$ and $I \subset S$ a homogeneous ideal of $S$ with $\operatorname{dim} S / I=d$. The Hilbert series $H_{S / I}(\lambda)$ of $S / I$ is of the form $H_{S / I}(\lambda)=\left(h_{0}+h_{1} \lambda+h_{2} \lambda^{2}+\cdots+h_{s} \lambda^{s}\right) /(1-\lambda)^{d}$, where each $h_{i} \in \mathbb{Z}$ ([2, Proposition 4.4.1]). We say that $h_{S / I}(\lambda)=h_{0}+h_{1} \lambda+h_{2} \lambda^{2}+\cdots+h_{s} \lambda^{s}$ with $h_{s} \neq 0$ is the h-polynomial of $S / I$. Let reg $(S / I)$ denote the (Castelnuovo-Mumford) regularity [2, p. 168] of $S / I$. A well-known fact (e.g., [3, Lemma 2.5]) is that, when $S / I$ is Cohen-Macaulay, one has

$$
\operatorname{reg}(S / I)=\operatorname{deg} h_{S / I}(\lambda) .
$$

Its converse is false. The following example was found by Jürgen Herzog. Let $I \subset$ $S=K\left[x_{1}, x_{2}, x_{3}, x_{4}\right]$ be the monomial ideal $\left(x_{2}^{3}, x_{2}^{2} x_{3}, x_{2} x_{3}^{2}, x_{3}^{3}, x_{1}^{2}, x_{1} x_{2}, x_{1} x_{3}, x_{1} x_{4}\right)$, which is strongly stable ([7, p. 103]). Then $\operatorname{dim} S / I=1, \operatorname{depth} S / I=0, \operatorname{reg}(S / I)=2$ and $h_{S / I}(\lambda)=$ $1+3 \lambda+2 \lambda^{2}$. At this stage, it is reasonable to discover a natural class of monomial ideals $I \subset S=K\left[x_{1}, \ldots, x_{n}\right]$ for which $S / I$ is not Cohen-Macaulay with $\operatorname{reg}(S / I)=\operatorname{deg} h_{S / I}(\lambda)$.

Furthermore, one cannot escape from the temptation to present the following

Conjecture 0.1. Given arbitrary integers $r$ and $s$ with $r \geq 1$ and $s \geq 1$, there exists a strongly stable ideal $I$ of $S=K\left[x_{1}, \ldots, x_{n}\right]$ with $n \gg 0$ for which $\operatorname{reg}(S / I)=r$ and $\operatorname{deg} h_{S / I}(\lambda)=s$.

It follows from [2, Lemma 4.1.3] that if $I$ has a pure resolution ([2, p. 153]), then

$$
\operatorname{deg} h_{S / I}(\lambda)-\operatorname{reg}(S / I)=\operatorname{dim} S / I-\operatorname{depth} S / I .
$$

2010 Mathematics Subject Classification. 05E40, 13H10.

Key words and phrases. Castelnuovo-Mumford regularity, $h$-polynomial, Cameron-Walker graph. 
As a result, when $1 \leq r \leq s$, a desired ideal can be found in the class of squarefree lexsegment ideals ([1], [7, p. 124]).

The purpose of the present paper is to give an affirmative answer to a weak version of Conjecture 0.1, that is to say, a monomial ideal $I$ of $S=K\left[x_{1}, \ldots, x_{n}\right]$ for which $\operatorname{reg}(S / I)=$ $r$ and $\operatorname{deg} h_{S / I}(\lambda)=s$ will be constructed.

Theorem 0.2. Given arbitrary integers $r$ and $s$ with $r \geq 1$ and $s \geq 1$, there exists a monomial ideal I of $S=K\left[x_{1}, \ldots, x_{n}\right]$ with $n \gg 0$ for which $\operatorname{reg}(S / I)=r$ and $\operatorname{deg} h_{S / I}(\lambda)=s$.

When $r>s$, a basic process in order to obtain a required ideal of Theorem 0.2 is to find a monomial ideal $I=I_{N} \subset S$ with $\operatorname{reg}(S / I)=N+1$ and $\operatorname{deg} h_{S / I}(\lambda)=1$ for an arbitrary integer $N>0$. A proof of Theorem 0.2 will be achieved in Section 1 .

On the other hand, in Section 2, we give a class of edge ideals $I \subset S$ of Cameron-Walker graphs ([8]) with $\operatorname{reg}(S / I)=\operatorname{deg} h_{S / I}(\lambda)$ for which $S / I$ is not Cohen-Macaulay.

\section{Proof of TheOrem 0.2}

Before giving a proof of Theorem 0.2 , several lemmata will be prepared. Let, as before, $S=K\left[x_{1}, \ldots, x_{n}\right]$ denote the polynomial ring in $n$ variables over a field $K$ with each $\operatorname{deg} x_{i}=1$. Lemma 1.1 below follows immediately from the definition of regularity in terms of graded Betti numbers ([7, p. 48]).

Lemma 1.1. Let $I \subset S$ be a proper homogeneous ideal. Then $\operatorname{reg}(S / I)=\operatorname{reg}(I)-1$.

Lemma 1.2 ([9, Lemma 3.2]). Let $S_{1}=K\left[x_{1}, \ldots, x_{m}\right]$ and $S_{2}=K\left[y_{1}, \ldots, y_{n}\right]$ be polynomial rings over a field $K$. Let $I_{1}$ be a nonzero homogeneous ideal of $S_{1}$ and $I_{2}$ that of $S_{2}$. Write $S$ for $S_{1} \otimes_{K} S_{2}=K\left[x_{1}, \ldots, x_{m}, y_{1}, \ldots, y_{n}\right]$ and regard $I_{1}+I_{2}$ and $I_{1} I_{2}$ as homogeneous ideals of $S$. Then

(1) $\operatorname{reg}\left(I_{1} I_{2}\right)=\operatorname{reg}\left(I_{1}\right)+\operatorname{reg}\left(I_{2}\right)$;

(2) $\operatorname{reg}\left(I_{1}+I_{2}\right)=\operatorname{reg}\left(I_{1}\right)+\operatorname{reg}\left(I_{2}\right)-1$;

(3) $\operatorname{reg}\left(S /\left(I_{1}+I_{2}\right)\right)=\operatorname{reg}\left(S_{1} / I_{1}\right)+\operatorname{reg}\left(S_{2} / I_{2}\right)$.

Lemma 1.3 ([6, Lemma 2.10]). Let I $\subset$ S be a monomial ideal and $x_{i}$ a variable of $S$ which appears in a monomial belonging to the unique minimal system of monomial generators of I. Then

$$
\operatorname{reg}(I) \leq \max \{\operatorname{reg}(I:(x))+1, \operatorname{reg}(I+(x))\} .
$$

In the first step of a proof of Theorem 0.2 , given integers $1 \leq r \leq s$, we construct a monomial ideal $I \subset S$ with $\operatorname{reg}(S / I)=r$ and $h_{S / I}(\lambda)=s$. As was mensioned in Introduction, a desired ideal $I$ can be found in the class of squarefree lexsegment ideals.

Let $<_{\text {lex }}$ denote the lexicographic order ([7, p. 24]) on $S=K\left[x_{1}, \ldots, x_{n}\right]$ induced from $x_{1}>x_{2}>\cdots>x_{n}$. A monomial ideal $I \subset S$ is called squarefree lexsegment if $I$ is generated by squarefree monomials and if, for all squarefree monomials $u \in I$ and for all squarefree monomials $v \in S$ with $\operatorname{deg} u=\operatorname{deg} v$ and $u<_{\operatorname{lex}} v$, one has $v \in I$. 
Fix integers $r$ and $s$ with $1 \leq r \leq s$ and consider the squarefree lexsegment ideal

$$
I_{r, s}=\left(u_{1} u_{2} \cdots u_{r} u_{r+1}, u_{1} u_{2} \cdots u_{r} u_{r+2}, \ldots, u_{1} u_{2} \cdots u_{r} u_{s+1}\right)
$$

of the polynomial ring $K\left[u_{1}, \ldots, u_{s+1}\right]$ in $(s+1)$ variables over a field $K$.

Proposition 1.4. One has

(1) $\operatorname{reg}\left(K\left[u_{1}, \ldots, u_{s+1}\right] / I_{r, s}\right)=r$;

(2) $H_{K\left[u_{1}, \ldots, u_{s+1}\right] / I_{r, s}}(\lambda)=\frac{1+\lambda+\cdots+\lambda^{r-1}+\lambda^{r}(1-\lambda)^{s-r}}{(1-\lambda)^{s}}$.

Thus, in particular, $\operatorname{deg} h_{K\left[u_{1}, \ldots, u_{s+1}\right] / I_{r, s}}(\lambda)=s$.

Proof. (1) Since $I_{r, s}=\left(u_{1} u_{2} \cdots u_{r}\right)\left(u_{r+1}, \ldots, u_{s+1}\right)$, it follows from Lemma 1.2 (1) that $\operatorname{reg}\left(I_{r, s}\right)=r+1$. Hence Lemma 1.1 says that $\operatorname{reg}\left(K\left[u_{1}, \ldots, u_{s+1}\right] / I_{r, s}\right)=r$, as desired.

(2) Let $u=u_{1} u_{2} \cdots u_{r}$. Then $I_{r, s}+(u)=(u)$ and $I_{r, s}:(u)=\left(u_{r+1}, \ldots, u_{s+1}\right)$. Thus the short exact sequence

$$
0 \rightarrow \frac{K\left[u_{1}, \ldots, u_{s+1}\right]}{I_{r, s}:(u)}(-r) \stackrel{\times u}{\longrightarrow} \frac{K\left[u_{1}, \ldots, u_{s+1}\right]}{I_{r, s}} \rightarrow \frac{K\left[u_{1}, \ldots, u_{s+1}\right]}{I_{r, s}+(u)} \rightarrow 0,
$$

yields

$$
\begin{aligned}
H_{K\left[u_{1}, \ldots, u_{s+1}\right] / I_{r, s}}(\lambda) & =H_{K\left[u_{1}, \ldots, u_{s+1}\right] /(u)}(\lambda)+\lambda^{r} \cdot H_{K\left[u_{1}, \ldots, u_{s+1}\right] /\left(u_{r+1}, \ldots, u_{s+1}\right)}(\lambda) \\
& =\frac{1+\lambda+\cdots+\lambda^{r-1}}{(1-\lambda)^{s}}+\frac{\lambda^{r}}{(1-\lambda)^{r}} \\
& =\frac{1+\lambda+\cdots+\lambda^{r-1}+\lambda^{r}(1-\lambda)^{s-r}}{(1-\lambda)^{s}}
\end{aligned}
$$

as required.

Proposition 1.4 guarantees that, when $1 \leq r \leq s$, Conjecture 0.1 is true.

Now, in the second step of a proof of Theorem 0.2 , we turn to the discussion of finding a desired monomial ideal for $1 \leq s<r$.

Let $n \geq 2$ and

$$
S_{n}=K\left[x, y_{1}, \ldots, y_{n}, z_{1}, \ldots, z_{n+1}\right]
$$

the polynomial ring in 2(n+1) variables over a field $K$. We then introduce the monomial ideals $I_{n}, J_{n}, K_{n}$ and $L_{n}$ defined as follows: 


$$
\begin{aligned}
I_{n}= & \left(x y_{1} y_{2} \cdots y_{n}, x z_{1} z_{2} \cdots z_{n+1}\right)+\sum_{i=1}^{n-2}\left(y_{1}, \ldots, y_{i}, y_{i+1} \cdots y_{n}\right)\left(z_{i}\right) \\
& +\left(y_{1}, \ldots, y_{n}\right)\left(z_{n-1}, z_{n}, z_{n+1}\right), \\
J_{n}= & \left(x z_{1} z_{2} \cdots z_{n+1}\right)+\sum_{i=1}^{n-1}\left(z_{i}, \ldots, z_{n+1}\right)\left(y_{i}\right), \\
K_{n}= & \left(x y_{1} y_{2} \cdots y_{n-1}\right)+\sum_{i=1}^{n-2}\left(y_{1}, \ldots, y_{i}, y_{i+1} \cdots y_{n-1}\right)\left(z_{i}\right), \\
L_{n}= & \sum_{i=1}^{n-1}\left(z_{i}, \ldots, z_{n}\right)\left(y_{i}\right) .
\end{aligned}
$$

Remark 1.5. One has

(1) $I_{n}+\left(y_{n}\right)=\left(y_{n}\right)+J_{n}$;

(2) $I_{n}:\left(y_{n}\right)=\left(z_{n-1}, z_{n}, z_{n+1}\right)+K_{n}$;

(3) $J_{n}+\left(z_{n+1}\right)=\left(z_{n+1}\right)+L_{n}$;

(4) $J_{n}:\left(z_{n+1}\right)=\left(x z_{1} z_{2} \cdots z_{n}\right)+\left(y_{1}, \ldots, y_{n-1}\right)$;

(5) $K_{n}+\left(y_{n-1}\right)=\left(y_{n-1}\right)+\sum_{i=1}^{n-2}\left(y_{1}, \ldots, y_{i}\right)\left(z_{i}\right)$ if $n \geq 3$;

(6) $K_{n}:\left(y_{n-1}\right)=\left(z_{n-2}\right)+K_{n-1}$ if $n \geq 3$;

(7) $L_{n}$ can be regarded as the edge ideal ([7, p. 156]) of the Ferrers graph associated with the partition $(n-1, n-1, n-2, \ldots, 2,1)$ and $\sum_{i=1}^{n-2}\left(y_{1}, \ldots, y_{i}\right)\left(z_{i}\right)$ appearing in (5) can be regarded as that with the partition $(n-2, n-1, \ldots, 2,1))$, see [4]. By using [4, Theorem 2.1], one has

$$
\begin{gathered}
\operatorname{reg}\left(L_{n}\right)=\operatorname{reg}\left(\sum_{i=1}^{n-2}\left(y_{1}, \ldots, y_{i}\right)\left(z_{i}\right)\right)=2, \\
H_{K\left[y_{1}, \ldots, y_{n-1}, z_{1}, \ldots, z_{n}\right] / L_{n}}(\lambda)=\frac{1+(n-1) \lambda-(n-1) \lambda^{2}}{(1-\lambda)^{n}}, \\
H_{K\left[y_{1}, \ldots, y_{n-2}, z_{1}, \ldots, z_{n-2}\right] / \sum_{i=1}^{n-2}\left(y_{1}, \ldots, y_{i}\right)\left(z_{i}\right)}(\lambda)=\frac{1+(n-2) \lambda}{(1-\lambda)^{n-2}} .
\end{gathered}
$$

Lemma 1.6. One has

(1) $\operatorname{reg}\left(J_{n}\right)=n+2$;

(2) $H_{K\left[x, y_{1}, \ldots, y_{n-1}, z_{1}, \ldots, z_{n+1}\right] / J_{n}}(\lambda)=\frac{1+n \lambda-(n-2) \lambda^{2}+\lambda^{3}+\cdots+\lambda^{n+1}}{(1-\lambda)^{n+1}}$. 
Proof. (1) Since $x z_{1} z_{2} \cdots z_{n+1}$ belongs to the unique minimal system of monomial generators of $J_{n}$, it follows that $n+2 \leq \operatorname{reg}\left(J_{n}\right)$. We claim $\operatorname{reg}\left(J_{n}\right) \leq n+2$. By using Lemma 1.2 (2) together with Remark 1.5 (3) and (7), one has

$$
\left.\operatorname{reg}\left(J_{n}+\left(z_{n+1}\right)\right)=\operatorname{reg}\left(\left(z_{n+1}\right)+L_{n}\right)\right)=\operatorname{reg}\left(L_{n}\right)=2 .
$$

Furthermore, Lemma 1.2 (2) together with Remark 1.5 (4) says that $\operatorname{reg}\left(J_{n}:\left(z_{n+1}\right)\right)=$ $n+1$. Hence, $\operatorname{reg}\left(J_{n}\right) \leq n+2$ follows from Lemma 1.3 .

(2) Let $T=K\left[x, y_{1}, \ldots, y_{n-1}, z_{1}, \ldots, z_{n+1}\right]$ and consider the short exact sequence

$$
0 \rightarrow \frac{T}{J_{n}:\left(z_{n+1}\right)}(-1) \stackrel{\times z_{n+1}}{\longrightarrow} \frac{T}{J_{n}} \rightarrow \frac{T}{J_{n}+\left(z_{n+1}\right)} \rightarrow 0 .
$$

Remark 1.5 (3) and (7) yield

$$
\begin{aligned}
H_{T / J_{n}+\left(z_{n+1}\right)}(\lambda) & =H_{T /\left(z_{n+1}\right)+L_{n}}(\lambda) \\
& =H_{K\left[y_{1}, \ldots, y_{n-1}, z_{1}, \ldots, z_{n}\right] / L_{n}}(\lambda) \cdot \frac{1}{(1-\lambda)} \\
& =\frac{1+(n-1) \lambda-(n-1) \lambda^{2}}{(1-\lambda)^{n+1}} .
\end{aligned}
$$

Furthermore, Remark 1.5 (4) yeilds

$$
\begin{aligned}
H_{T / J_{n}:\left(z_{n+1}\right)}(\lambda) & =H_{T /\left(x z_{1} z_{2} \cdots z_{n}\right)+\left(y_{1}, \ldots, y_{n-1}\right)}(\lambda) \\
& =H_{K\left[x, z_{1}, \cdots, z_{n}\right] /\left(x z_{1} z_{2} \cdots z_{n}\right)}(\lambda) \cdot \frac{1}{(1-\lambda)} \\
& =\frac{1+\lambda+\cdots+\lambda^{n}}{(1-\lambda)^{n+1}} .
\end{aligned}
$$

Hence

$$
\begin{aligned}
H_{T / J_{n}}(\lambda) & =H_{T / J_{n}+\left(z_{n+1}\right)}(\lambda)+\lambda \cdot H_{T / J_{n}:\left(z_{n+1}\right)}(\lambda) \\
& =\frac{1+n \lambda-(n-2) \lambda^{2}+\lambda^{3}+\cdots+\lambda^{n+1}}{(1-\lambda)^{n+1}}
\end{aligned}
$$

as desired.

\section{Lemma 1.7. One has}

(1) $\operatorname{reg}\left(K_{n}\right)=n$;

(2) $H_{K\left[x, y_{1}, \ldots, y_{n-1}, z_{1}, \ldots, z_{n-2}\right] / K_{n}}(\lambda)=\frac{1+\sum_{i=1}^{n-1}(n-i) \lambda^{i}}{(1-\lambda)^{n-1}}$. 
Proof. Since $x y_{1} y_{2} \cdots y_{n-1}$ belongs to the unique minimal system of monomial generators of $J_{n}$, one has $n \leq \operatorname{reg}\left(K_{n}\right)$. We claim $\operatorname{reg}\left(K_{n}\right) \leq n$ and (2) by using induction on $n$. Since $K_{2}=\left(x y_{1}\right)$, each of the assertion is trivial for $n=2$. Let $n>2$. Lemma 1.2 (2) together with Remark 1.5 (5) and (7) guarantees that

$$
\operatorname{reg}\left(K_{n}+\left(y_{n-1}\right)\right)=\operatorname{reg}\left(\left(y_{n-1}\right)+\sum_{i=1}^{n-2}\left(y_{1}, \ldots, y_{i}\right)\left(z_{i}\right)\right)=2 .
$$

Moreover, by virtue of Lemma[1.2](2), Remark[1.5(6) as well as the induction hypothesis, it follows that

$$
\operatorname{reg}\left(K_{n}:\left(y_{n-1}\right)\right)=\operatorname{reg}\left(\left(z_{n-2}\right)+K_{n-1}\right)=\operatorname{reg}\left(K_{n-1}\right)=n-1 .
$$

Hence, Lemma 1.3 says that $\operatorname{reg}\left(K_{n}\right) \leq n$.

Now, consider the short exact sequence

$$
0 \rightarrow \frac{T^{\prime}}{K_{n}:\left(y_{n-1}\right)}(-1) \stackrel{\times y_{n-1}}{\longrightarrow} \frac{T^{\prime}}{K_{n}} \rightarrow \frac{T^{\prime}}{K_{n}+\left(y_{n-1}\right)} \rightarrow 0,
$$

where $T^{\prime}=K\left[x, y_{1}, \ldots, y_{n-1}, z_{1}, \ldots, z_{n-2}\right]$. It follows from Remark 1.5](5) and (7) that

$$
\begin{aligned}
H_{T^{\prime} / K_{n}+\left(y_{n-1}\right)}(\lambda) & =H_{T^{\prime} /\left(y_{n-1}\right)+\sum_{i=1}^{n-2}\left(y_{1}, \ldots, y_{i}\right)\left(z_{i}\right)}(\lambda) \\
& =H_{K\left[y_{1}, \ldots, y_{n-2}, z_{1}, \ldots, z_{n-2}\right] / \Sigma_{i=1}^{n-2}\left(y_{1}, \ldots, y_{i}\right)\left(z_{i}\right)}(\lambda) \cdot \frac{1}{1-\lambda} \\
& =\frac{1+(n-2) \lambda}{(1-\lambda)^{n-1}} .
\end{aligned}
$$

Furthermore, Remark 1.5 (6) as well as the induction hypothesis guarantees that

$$
\begin{aligned}
H_{T^{\prime} / K_{n}:\left(y_{n-1}\right)}(\lambda) & =K_{T^{\prime} /\left(z_{n-2}\right)+K_{n-1}}(\lambda) \\
& =H_{K\left[x, y_{1}, \ldots, y_{n-2}, z_{1}, \ldots, z_{n-3}\right] / K_{n-1}}(\lambda) \cdot \frac{1}{1-\lambda} \\
& =\frac{1+\sum_{i=1}^{n-2}(n-1-i) \lambda^{i}}{(1-\lambda)^{n-1}} .
\end{aligned}
$$


Hence, one has

$$
\begin{aligned}
H_{T^{\prime} / K_{n}}(\lambda) & =H_{T^{\prime} / K_{n}+\left(y_{n-1}\right)}(\lambda)+\lambda \cdot H_{T^{\prime} / K_{n}:\left(y_{n-1}\right)}(\lambda) \\
& =\frac{1+(n-2) \lambda+\lambda+\lambda \cdot \sum_{i=1}^{n-2}(n-1-i) \lambda^{i}}{(1-\lambda)^{n-1}} \\
& =\frac{1+(n-1) \lambda+\sum_{i=1}^{n-2}(n-1-i) \lambda^{i+1}}{(1-\lambda)^{n-1}} \\
& =\frac{1+(n-1) \lambda+\sum_{i=2}^{n-1}(n-i) \lambda^{i}}{(1-\lambda)^{n-1}} \\
& =\frac{1+\sum_{i=1}^{n-1}(n-i) \lambda^{i}}{(1-\lambda)^{n-1}}
\end{aligned}
$$

as desired.

The monomial ideal $I_{n}$ plays an important role in our proof of Theorem 0.2 .

\section{Proposition 1.8. One has}

(1) $\operatorname{reg}\left(S_{n} / I_{n}\right)=n+1$;

(2) $H_{S_{n} / I_{n}}(\lambda)=\frac{1+(n+1) \lambda}{(1-\lambda)^{n+1}}$.

Thus, in particular, $\operatorname{deg} h_{S_{n} / I_{n}}(\lambda)=1$.

Proof. (1) By virtue of Lemma 1.1, it is sufficient to show that $\operatorname{reg}\left(I_{n}\right)=n+2$. Since $x z_{1} z_{2} \cdots z_{n+1}$ belongs to the unique minimal system of monomial generators of $I_{n}$, one has $n+2 \leq \operatorname{reg}\left(I_{n}\right)$. We claim $\operatorname{reg}\left(I_{n}\right) \leq n+2$. It follows from Lemma 1.2 (2), Remark 1.5 (1) together with Lemma 1.6 (1) that

$$
\operatorname{reg}\left(I_{n}+\left(y_{n}\right)\right)=\operatorname{reg}\left(\left(y_{n}\right)+J_{n}\right)=\operatorname{reg}\left(J_{n}\right)=n+2 .
$$

By using Lemma1.2(2), Remark1.5(2) together with Lemma1.7(1), one has

$$
\operatorname{reg}\left(I_{n}:\left(y_{n}\right)\right)=\operatorname{reg}\left(\left(z_{n-1}, z_{n}, z_{n+1}\right)+K_{n}\right)=\operatorname{reg}\left(K_{n}\right)=n .
$$

Hence Lemma 1.3 says that $\operatorname{reg}\left(I_{n}\right) \leq n+2$, as desired.

(2) Considering the short exact sequence

$$
0 \rightarrow \frac{S_{n}}{I_{n}:\left(y_{n}\right)}(-1) \stackrel{\times y_{n}}{\longrightarrow} \frac{S_{n}}{I_{n}} \rightarrow \frac{S_{n}}{I_{n}+\left(y_{n}\right)} \rightarrow 0
$$


Remark 1.5 (1) together with Lemma 1.6 (2) yields

$$
\begin{aligned}
H_{S_{n} / I_{n}+\left(y_{n}\right)}(\lambda) & =H_{S_{n} /\left(y_{n}\right)+J_{n}}(\lambda) \\
& =H_{K\left[x, y_{1}, \ldots, y_{n-1}, z_{1}, \ldots, z_{n+1}\right] / J_{n}}(\lambda) \\
& =\frac{1+n \lambda-(n-2) \lambda^{2}+\lambda^{3}+\cdots+\lambda^{n+1}}{(1-\lambda)^{n+1}} .
\end{aligned}
$$

Furthermore, Remark 1.5 (2) together with Lemma 1.7(2) yields

$$
\begin{aligned}
H_{S_{n} / I_{n}:\left(y_{n}\right)}(\lambda) & =H_{S_{n} /\left(z_{n-1}, z_{n}, z_{n+1}\right)+K_{n}}(\lambda) \\
& =H_{K\left[x, y_{1}, \ldots, y_{n-1}, z_{1}, \ldots, z_{n-2}\right] / K_{n}}(\lambda) \cdot \frac{1}{1-\lambda} \\
& =\frac{1+\sum_{i=1}^{n-1}(n-i) \lambda^{i}}{(1-\lambda)^{n}} .
\end{aligned}
$$

It then follows that

$$
\begin{aligned}
& H_{S_{n} / I_{n}}(\lambda)=H_{S_{n} / I_{n}+\left(y_{n}\right)}(\lambda)+\lambda \cdot H_{S_{n} / I_{n}:\left(y_{n}\right)}(\lambda) \\
= & \frac{1+n \lambda-(n-2) \lambda^{2}+\lambda^{3}+\cdots+\lambda^{n+1}}{(1-\lambda)^{n+1}}+\frac{t+\sum_{i=1}^{n-1}(n-i) \lambda^{i+1}}{(1-\lambda)^{n}} \\
= & \frac{1+n \lambda-(n-2) \lambda^{2}+\lambda^{3}+\cdots+\lambda^{n+1}+\lambda(1-\lambda)+(1-\lambda) \sum_{i=1}^{n-1}(n-i) \lambda^{i+1}}{(1-\lambda)^{n+1}} \\
= & \frac{1+(n+1) \lambda-(n-1) \lambda^{2}+\sum_{i=2}^{n} \lambda^{i+1}+\sum_{i=1}^{n-1}(n-i) \lambda^{i+1}-\sum_{i=1}^{n-1}(n-i) \lambda^{i+2}}{(1-\lambda)^{n+1}} \\
= & \frac{1+(n+1) \lambda-(n-1) \lambda^{2}+\sum_{i=2}^{n} \lambda^{i+1}+\sum_{i=1}^{n-1}(n-i) \lambda^{i+1}-\sum_{i=2}^{n}(n-i+1) \lambda^{i+1}}{(1-\lambda)^{n+1}} \\
= & \frac{1+(n+1) \lambda-(n-1) \lambda^{2}+\sum_{i=2}^{n} \lambda^{i+1}+(n-1) \lambda^{2}-\sum_{i=2}^{n} \lambda^{i+1}}{(1-\lambda)^{n+1}} \\
= & \frac{1+(n+1) \lambda}{(1-\lambda)^{n+1}},
\end{aligned}
$$

as required.

We are now in the position to finish a proof of Theorem 0.2 . 
Proof. (Proof of Theorem 0.2 ) Let $r$ and $s$ be positive integers with $r, s \geq 1$. By virtue of Proposition 1.4, only the case of $r>s \geq 1$ will be discussed. Let $S_{1}=K\left[x, y_{1}, z_{1}, z_{2}\right]$ and $I_{1}=\left(x y_{1}, x z_{1} z_{2}, y_{1} z_{1}, y_{1} z_{2}\right)$. It then follows that $\operatorname{reg}\left(S_{1} / I_{1}\right)=2$ and $H_{S_{1} / I_{1}}(\lambda)=\frac{1+2 \lambda}{(1-\lambda)^{2}}$. By virtue of this example and of Proposition 1.8, one has

$$
\operatorname{reg}\left(S_{r-s} / I_{r-s}\right)=r-s+1, H_{S_{r-s} / I_{r-s}}(\lambda)=\frac{1+(r-s+1) \lambda}{(1-\lambda)^{r-s+1}} .
$$

Let $S=S_{r-s} \otimes_{K} K\left[u_{1}, \ldots, u_{s}\right]$ and $I=I_{r-s}+\left(u_{1} u_{2} \cdots u_{s}\right)$. Lemma 1.2 (3) together with Proposition 1.4 yields

$$
\operatorname{reg}(S / I)=r-s+1+s-1=r
$$

and

$$
H_{S / I}(\lambda)=\frac{\{1+(r-s+1) \lambda\}\left(1+\lambda+\cdots+\lambda^{s-1}\right)}{(1-\lambda)^{r}} .
$$

Hence $\operatorname{deg} h_{S / I}(\lambda)=s$ and $I$ is a desired monomial ideal.

\section{EXAMPLES}

The purpose of this section is to give a class of edge ideals $I \subset S$ of Cameron-Walker graphs $([\overline{8}])$ with $\operatorname{reg}(S / I)=\operatorname{deg} h_{S / I}(\lambda)$ for which $S / I$ is not Cohen-Macaulay.

Let $G$ be a finite simple graph on the vertex set $[n]=\{1, \ldots, n\}$ and $E(G)$ its edge set. (A finite graph is called simple if it possesses no loop and no multiple edge.) The edge ideal $I(G)$ of $G$ is the monomial ideal of $S=K\left[x_{1}, \ldots x_{n}\right]$ generated by those quadratic monomials $x_{i} x_{j}$ with $\{i, j\} \in E(G)$ :

$$
I(G)=\left(x_{i} x_{j}:\{i, j\} \in E(G)\right) \subset S .
$$

In general, it is quite difficult to compute the regularity of an edge ideal. However, one can compute $\operatorname{reg}(I(G))$ easily if $G$ is a Cameron-Walker graph. The notion of CameronWalker graph was introduced by [5]. We refer the reader to [8, p. 258] for a classification of Cameron-Walker graphs.

Example 2.1. Fix $m \geq 1$ and write $G_{m}^{1}$ for the star triangle joining $m$ triangles at one common vertex. Then $S / I\left(G_{m}^{1}\right)$ is Cohen-Macaulay if and only if $m=1$ ([8, Theorem 1.3]). Hence

- If $m=2 k$, then $\operatorname{reg}\left(S / I\left(G_{m}^{1}\right)\right)=2 k>2 k-1=\operatorname{deg} h_{S / I\left(G_{m}^{1}\right)}(\lambda)$.

- If $m=2 k+1$, then $\operatorname{reg}\left(S / I\left(G_{m}^{1}\right)\right)=\operatorname{deg} h_{S / I\left(G_{m}^{1}\right)}(\lambda)=2 k+1$. 
Example 2.2. Fix $m \geq 1$ and write $G_{m}^{2}$ for the graph drawn below on the vertex set $[2 m+$ 3]:

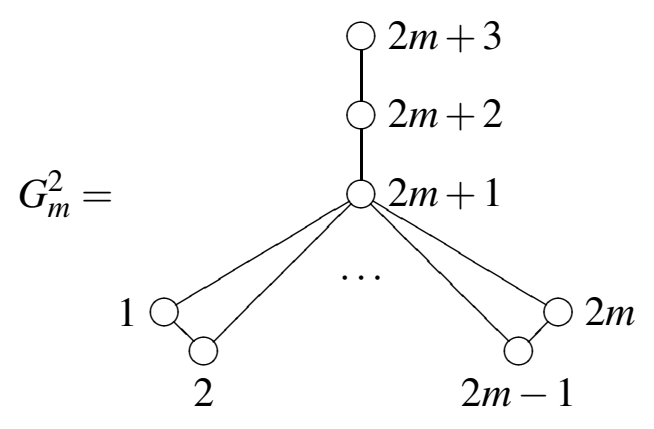

Then [8, Theorem 1.3] says that $G_{m}^{2}$ is not Cohen-Macaulay if $m \geq 2$. However,

$$
\operatorname{reg}\left(S / I\left(G_{m}^{2}\right)\right)=\operatorname{deg} h_{S / I\left(G_{m}^{2}\right)}(\lambda)=m+1 .
$$

On the other hand, this graph is of interest from the viewpoint of $h$-vector $\left(h_{0}, h_{1}, \ldots, h_{s}\right)$, which is the sequence of coefficients of $h$-polynomial. It is known [10, Theorem 4.4] that the $h$-vector of Gorenstein ring is symmetric, but the converse is false. A routine computation shows that

$$
h_{S / I\left(G_{m}^{2}\right)}(\lambda)=(1+\lambda)^{m+1}+\lambda(1-\lambda)^{m-1} .
$$

Hence $h$-vector of $S / I\left(G_{m}^{2}\right)$ is symmetric if $m$ is odd, but not necessary unimodal. For example, the $h$-vector of $S / I\left(G_{3}^{2}\right)$ is $(1,5,4,5,1)$, which is not unimodal. In general, the $h$-vector of $S / I\left(G_{m}^{2}\right)$ is not unimodal if $m=4 k+3$.

Acknowledgment. The first author was partially supported by JSPS KAKENHI 26220701. The second author was partially supported by JSPS KAKENHI 17K14165.

\section{REFERENCES}

[1] A. Aramova, J. Herzog and T. Hibi, Squarefree lexsegment ideals, Math. Z. 228 (1998), 353-378.

[2] W. Bruns and J. Herzog, Cohen-Macaulay Rings, Revised ED., Cambridge Stud. Adv. Math., vol. 39, Cambridge University Press, Cambridge, 1998.

[3] B. Benedetti and M. Varbaro, On the dual graph of Cohen-Macaulay algebras, Int. Math. Res. Not. IMRN 17 (2015), 8085-8115.

[4] A. Corso and U. Nagel, Monomial and toric ideals associated to Ferrers graphs, Trans. Amer. Math. Soc. 361 (2009), 1371-1395.

[5] K. Cameron and T. Walker, The graphs with maximum induced matching and maximum matching the same size, Discrete Math. 299 (2005), 49-55.

[6] H. Dao, C. Huneke and J. Schweig, Bounds on the regularity and projective dimension of ideals associated to graphs, J. Algebraic Combin. 38 (2013), 37-55.

[7] J. Herzog and T. Hibi, Monomial ideals, Graduate Texts in Mathematics 260, Springer, London, 2010. 
[8] T. Hibi, A. Higashitani, K. Kimura and A. B. O'Keefe, Algebraic study on Cameron-Walker graphs, J. of Algebra 422 (2015), 257-269.

[9] L. T. Hoa and N. D. Tam, On some invariants of a mixed product of ideals, Arch. Math. (Basel) 94 (2010), 327-337.

[10] R. Stanley, Hilbert functions of graded algebras, Adv. Math. 28 (1978), 57-83.

Takayuki Hibi, Department of Pure and Applied Mathematics, Graduate School of

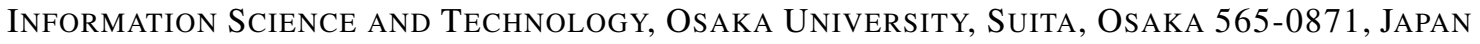

E-mail address: hibi@math.sci.osaka-u.ac.jp

Kazunori Matsuda, Department of Pure and Applied Mathematics, Graduate School OF INFORMATION SCIENCE AND TEChNOLOGY, OSAKA UNIVERSiTy, SUITA, OsAKa 565-0871, JAPAN

E-mail address: kaz-matsuda@ist.osaka-u.ac.jp 